\title{
A concept of a mainly digitalized course on control theory including problembased practical units and digital supported exams
}

\section{Birgit Rösel}

Fakulty of Electrical and Computer Engineering, OTH Regensburg - University of Applied Science, Germany.

\begin{abstract}
This paper decribes the concept of a course on control theory as a minor subject in the department of electrical engineering at the OTH Regensburg. The basis for a new concept is the idea of contructive alignment. The whole teaching module is devided in two blocks. The first block contains the teaching of the knowledge about control theory with an integrated approach for lectures, exercises and practical sessions. In the second block the students attent a practical work package to have the possibility to applicate and deepen their knowledge. To foster active participation the students are asked to document and reflect their work with the course context in an electronic portfolio. The exam of this module consists of three parts with respect to the different competencies obtained during the module.
\end{abstract}

This concept develops not only the knowledge on control theory but personal and social competencies which are of growing interest. The aim of the paper is to present the idea of the concept. Following papers will illustrate the development process and the results obtained later on.

Keywords: constructive alignment; integrated approach; knowledge based working unit; electronic portfolio; develop competencies. 


\section{Introduction}

A digitalized concept for teaching control theory as minor subject with an integrated approach for lectures, exercises and practical sessions in the department of electrical engineering at the OTH Regensburg was established by the author in winterterm 2016/17. The concept uses activating methods like blended learning and possibilities of digitalization of teaching implementing Just in Time Teaching and Peer Instruction. The basis of this concept is the idea of constructive alignment. [Köhler, Th., Rösel, B. (2019)]

In winterterm 2019/20 a new course of studies was found on the department of electrical engineering at the OTH Regensburg. This course contains control theory as well, but due to a different set of courses the control theory can be set up on a higher level. Thus the concept presented here is based on the experiences obtained with the concept shown in Köhler and Rösel (2019) but contains additionally a three-week problem based practical work package. Furthermore the students are requested to work with a digital portfolio to document their working results and reflect their own work. The whole module is worth seven credit points.

The exam of this modul consists of three parts - a pre-test based on a concept inventory on signals and systems, a classical theoretical exam where the usage of the digital portfolio as exclusive resource is allowed and finally the outcome of the practical work package.

\section{Teaching concept}

\subsection{Development of the concept}

The development of the concept for the new module of control theory started with the definition of learning objectives for the whole module based on the learning objectives of the module started in winterterm 2016/17 by taking into concideration what students already learned in the first three terms of their studies. It was an aim to achieve a strong relationship between theoretical concepts and their practical relevance. Consequently there are lessons to impart theoretical knowledge, exercise units to recognize the practical use of this knowledge and practical work units to improve the understanding of the theory. To achieve that aim a structure for the whole teaching module was created giving a defined sequence of lessons, exercises and practical work shown in figure 1. This structure formed the basis for the acquisition of learning objectives for every lecture, classified according to the taxonomy of Biggs (2003). Furthermore each learning objective was associated with one of the following aspects: technical, methodical, social or personal.

According to the idea of constructive alignment the classification of learning outcomes was important for the decision which content should be dealt with which didactic approach. 
To foster active participation during the whole module the students are asked to document and reflect their work with the course context in an electronic portfolio.

The whole teaching module is divided into two blocks - the teaching of the knowledge about control theory with an integrated approach for lectures, exercises and practical sessions and the application and deeper routing of this knowledge with a problem based practical work package. Both blocks consist of several units.

The first two units of the module are used to explain the didactic concepts, to make the participants familiar with the digital portfolio and to do the pre-test. Refering to Figure 1, this two units are marked with greek letters.

First block

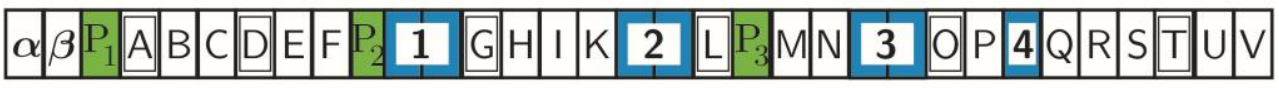

Second block

Exam

practical work package

Figure 1. Structure of the whole teaching module with lessons (latin letters, with frame-blended learning unit), exercises (numbers on blue ground), practical work units (PX on green ground) and the problem based practical work package. Each frame represents on session of 90 minutes.

\subsection{Integrated approach for lectures, exercises and practical sessions}

The first block of the module adopts the concept as described in Rösel and Köhler (2018) and Köhler and Rösel (2019). This concept uses activating methods like blended learning and possibilities of digitalization of teaching implementing Just in Time Teaching and Peer Instruction. Just in Time Teaching or JiTT is a strategy based on several principles of pedagogical best practice. In the concept described in this paper, students must read a specific teaching text before a blended learning unit. Furthermore, it is required to answer several questions regarding the content of the text. The decision which content is taught with a JiTT strategy depends on the level of learning objective in a technical or methodical aspect. The highest level of learning objective was the essential criterion for this decision. That is why the most difficult content can be specifically adopted by the lecturer to each group and discussed during the attendance phase of the blended learning unit. Thus students attend class well prepared and the lecturer is primed for this particular group of students while attending class, as stated by Gavrin (2006).

After more than four terms experience with this integrated approach it can be stated that participants complain about the higher effort at the beginning of the semester. But then up to $80 \%$ read and work through all teaching texts or leave out only one text. Teaching evaluation 
shows an substantial improvement especially regarding the categories "interest for the course" and "increase in knowledge". The self-assessment of the students shows furthermore that they feel more competent regarding methodical and professional competencies which leads to a higher satisfaction of the students regarding the teaching method.

The teaching texts were written by the author of this paper based on several approved literatur for control theory. By that the possibility of adopting the texts to the specific needs of this particular module is given and additionally students have the opportunity to become familiar with different textbooks. Each teaching text has the same structure and is roughly six to eight pages long. The teaching texts are given to the participants at a dedicated eLearning platform of our university. This starts the online phase of a blended learning unit. The students need to read the text during their self-learning time before the attendance phase. Furthermore, they must answer questions belonging to the text during the online phase. The answers have to be delivered at least 24 hours before the attendance phase starts by using the eLearning platform.

There are questions testing a basic understanding of the content as well as conceptual questions or exercises. Furthermore there is an open question asking if there are still issues outstanding. If this is the case, the participants may ask their question. If there is no issue open, they shall give a statement on the most important message in the text. The evaluation of this open question from the students must be done by the lecturer. The attendance phase starts with a summary of the correct answers of the questions given to the students. But the questions and statements from them to the final open question form the main part. The questions raised by the students present a new viewpoint for the lecturer, providing the possibility to explain what the participants really want to know and not what the lecturer thinks they may need. As a result, the number of participants in the attendance phase is equal or even higher than in classical lectures. The attendance phase may end with additional conceptual questions to be discussed in class using Peer Instruction. Peer Instruction or PI is an interactive teaching technique that promotes classroom interaction and thus complements the idea of JiTT, as stated by Watkins and Mazur (2010).

In the concept presented in this paper not every communication of theoretical knowledge is done by a blended learning attempt, as there are classical lectures as well. The decision which content is provided with which didactic approach depends on the level of classification of the learning outcomes. Based on four terms of experience a ratio of 3 to 1 of classical lectures and blended learning units seems to be an optimum regarding workload for students and lecturer. Refering to Figure 1, lessons are marked with a letter, the blended learning units have an additional frame.

The exercise units are placed on the end of a certain theoretical chapter to recognize the practical use of the content discussed in this chapter. There are tasks given to be prepared by the students and to be discussed during the exercise units. To ensure enough time to discuss 
the tasks there are two successive exercise units planed for some chapters. Refering to Figure 1 , the exercise units are marked with numbers on blue ground.

There are three practical work units during the first block of the module. Refering to Figure 1 , this practical work units are marked with " $\mathrm{P}_{\mathrm{X}}$ " on green ground. This units take place during a normal teaching unit of 90 minutes focusing on one specific aspect discussed in the lectures before. The aim of these units is to improve the understanding and to recognise possible deviations between theory taught and the practical behaviour of systems. As the aim is a deeper understanding of the topics discussed in the lectures before there is no obligation to attend these units. The feedback of the students regarding this integrated approach is very good. Normally, more then $80 \%$ of the attendants of the lectures participate the practical work units as well.

\subsection{Problem based practical work package}

The first block is followed by a second one, a set of problem based practical work units covering three weeks during the semester. As there are three lectures per week within this module there are nine lectures to work on the problem. Refering to Figure 1, this practical work package is marked as one block on green ground.

The students work together in groups of up to six participants. First, they choose a system out of a set of possibilities given by the lecturer. The systems are mainly real objects such as small vehicles, water tanks, inverted pendulum, not simulation models. The task is to analyze the system and to develop a control for it. The concrete formulation of the task of the own group is the first challenge in this work package. The aim is not necessarily a proper control but three weeks of intensive work with the system choosen. Maybe one group is working hard on a proper model for its system so the design of the control might be less challenging. Another group might use a given model and focuses on different control strategies and their advantages and disadvantages. Thus, the students review the control theory given to them in the first block and try to solve a real problem with restrictions as they will have in their future life as electrical engineers.

The participants have to define and describe the task they plan to solve using the digital portfolio. The steps they do in the development process of the control has to be documented and reflected in this portfolio as well. Finally, they have to present their task and results to their peers by a presentation. Thus during the second block the students are forced to use the knowledge abtained in the first block. Additionally, they develop social and personal competencies which are essential for their future work. 


\section{Concept for the exams}

The exam of this module consists of three parts with respect to the different competencies obtained during the module. That is a test based on a concept inventory on signals and systems, a classical theoretical exam where the usage of the digital portfolio as exclusive resource is allowed and finally the outcome of the practical work package. All three parts are mandatory to succeed in order to pass the whole module.

\subsection{Concept inventory}

As the base of control theory is signal and system theory it is obvious to define knowledge about signal and system theory as a prerequisite for a module on control theory. Thus it is planned to use a concept inventory on this field of knowledge.

Concept inventories measure the understanding of concepts rather than the ability to solve computational tasks by standardized tests. This method is well known and quite often used in the US but still relatively unknown in Germany. There is a concept inventory on signals and systems developed by Wage et.al. (2005), containing exactly the concepts needed for control theory. The author plans to apply this concept inventory to confront the students with their level of knowledge at the beginning of the module and to sensitize them to the need of this knowledge. The results obtained by this concept inventory make up $10 \%$ of the overall result.

\subsection{Exam with digital support}

The main part of the overall results are a classical theoretical exam on control theory examing the ability of the students to choose the right method to describe and solve a control problem by processing conceptual and computational tasks. This exam will take place after the three weeks practical work unit (refer to Figure 1). Thus students were able to consolidate and deepen their theoretical knowledge. This exam determines the overall results as it has a $60 \%$ share of it.

The tasks will be given on paper and the students have to work on it. But in contrary to other exams the usage of the digital portfolio as exclusive resource provided by an electronic device is allowed. The students are asked to document their learning process in a personal digital portfolio. This portfolio might content all teaching texts and the related answered questions to each text as well as all tasks concerning control theory worked on during the whole module and processed parts of the script. Thus the personal portfolio shall be structured and developed by each student according to his or her personal need in order to give the best support during the exam. 


\subsection{Presentation of the results of the practical work package}

The student groups working together on one control task during the practical work package shall present their self-imposed task and the results obtained to their peers. Therefore they shall create a special site in their digital portfolio. This site and the presentation will be evaluated using a rubric. A rubric is a evaluation matrix where several aspects of a task can be marked in a range of points. The outcome is a number of points as a sum of all aspects.

To improve personal and social competencies this rubric will be filled out not only by the lecturer but by students as well. This will be done at the last unit of the practical work units. The actual grading will be given by the lecturer as a mean value of the rubrics. This third part of the exam has a $30 \%$ share of the overall result.

\section{Implementation plan and expectations}

The first ideas regarding this concept were developed during the setup of the new course of studies. Based on the concept presented in this paper the details as learning texts, working and examination documents will be worked out during summer term 2020. Additionally, some technical questions regarding the digital portfolio and its usage in an exam have to be answered. In winter term 2020/21 some parts of the concept will be evaluated and tested with volunteers and finally in summer term 2021 the module will be started for the first time.

The new concept described in this paper is based on the experiences with the integrated approach described in Rösel and Köhler (2018) and Köhler and Rösel (2019). The author expects that students accept the additional workload because they recognize the positive effect of continuous work during the semester. The problem based practical work package simulate a real life problem leading to a deeper understanding of control theory by directly implementing it. Furthermode the module improves social and personal competencies of the students which are essential for their future work.

As usual the participation of each student on every lesson is not mandatory but of course highly recommended. The author expects a high participation rate and satisfaction with the module as students normally appreciate courses giving them the possibility to work like in their future job life.

\section{References}

Biggs, J. (2003). Aligning teaching and assessing to course objectives. Teaching and Learning in Higher Education: New Trends ans Innovations, 2003.

Gavrin, A. (2006). Just-in-time teaching. Metropolitan Universities 17(4), pp. 9-18, 2006. 
Köhler, Th., Rösel, B. (2019). Experiences with a new Digitalized Concept for Teaching Control Theory as Minor Subject at a University of Applied Science. 2019 IEEE Global Engineering Education Conference (EDUCON). doi: 10.1109/EDUCON.2019.8725217

Rösel, B., Köhler, Th. (2018). First results of a new digitalized concept for teaching control theory as minor subject at a university of applied science. 2018 IEEE Global Engineering Education Conference (EDUCON). doi: 10.1109/EDUCON.2018.8363217

Wage, Kathleen E., Buck, John R., Wright, Cameron H. G., Welch, Thad B. (2005). The Signals and Systems Concept Inventory. IEEE TRANSACTIONS ON EDUCATION, VOL. 48, NO. 3, AUGUST 2005, pp. 448-461.

Watkins, J., Mazur, E. (2010). Just-in Time Teaching and Peer Instruction. Just-in Time Teaching, 2010, pp. 39-62. 